\title{
PREPARING FOR CLINICAL DRIFT OF PET/CT, AN EXAMPLE FROM NORWAY
}

\author{
Julie Haglund* \\ Medical Physicist, Göteborg, Sweden
}

\begin{abstract}
The challenge of installing and starting clinical operations of PET/CT in a hospital requires cooperation from a multidisciplinary team. When not only the PET/CT modality is new to the department, but also the entire hospital facility is new, even greater efforts are required in order to successfully begin clinical activity. At the new Østfold Hospital Kalnes in Norway, the decision to install a PET/CT in the nuclear medicine department was made after designing and planning a room for SPECT/CT, which meant modifying the physical buildings of the new hospital during a late phase of construction. Shielding had to be increased in order to accommodate positron annihilation radiation energy. Østfold Hospital Kalnes had the opportunity to choose among three manufacturers of PET/CT machines, which required evaluation and research by a multidisciplinary team in order to meet the present and future ambitions of the hospital. The staff of the nuclear medicine department worked with clinical SPECT/CT, but nobody had previously worked with PET/CT. Training became the critical part of preparing for the installation and clinical implementation of a new modality. It was also decided to join the EARL FDG quality assurance program from the European Association of Nuclear Medicine. Participation in EARL enhances confidence of staff and patients, and increases possibilities for collaboration and research.
\end{abstract}

Key words: PET/CT purchase, Sykehuset Østfold, Kalnes, PET/CT tenure, PET shielding

DOI: $10.21175 / \operatorname{RadProc} .2016 .25$

\section{INTRODUCTION}

As described by the AAPM Task Group 108 and IAEA, the challenge of installing and starting clinical operations of PET/CT in a hospital requires cooperation from a multidisciplinary team $[1,2]$. When not only the PET/CT modality is new to the department, but also the entire hospital facility is new, even greater efforts are required in order to successfully begin clinical activity. At the new Østfold Hospital Kalnes in Norway, the decision to install a PET/CT in the nuclear medicine department required starting from scratch in every possible way. This work focuses on the shielding, procurement, and training activities that took place prior to installation.

The nuclear medicine department was designed and constructed for two SPECT/CT machines. The decision to operate PET/CT in one of the rooms required modifying the newly constructed department to accommodate the energy of positron annihilation radiation. Østfold Hospital Kalnes had the opportunity to choose among three manufacturers of PET/CT machines which system to purchase. The decision had to suit both the present limitations and future ambitions of the hospital. The staff of the nuclear medicine department is well experienced in clinical SPECT/CT, but nobody had previously worked with PET/CT. Training the staff became a critical part of preparing for the installation and clinical implementation of a new modality.

*haglundj@aol.com

\section{MATERIALS AND METHODS}

\subsection{Shielding}

Østfold Hospital Kalnes was an entirely new construction that began in 2011 and was completed in autumn 2015. During planning and building, the nuclear medicine department was designed and constructed for two SPECT/CT machines. One SPECT/CT would be moved from the old hospital and another would be purchased, but late changes in budget allowed for the purchase of a PET/CT instead of an additional SPECT/CT. Turning a purchasing desire into a realistic decision required considering what changes are necessary when installing PET/CT instead of SPECT/CT. One of the rooms allotted for SPECT/CT had to be changed in order to meet the shielding requirements and workflow patterns of clinical PET/CT operations.

National guidelines dictate radiation exposure limits for controlled and uncontrolled areas, and a physicist calculated the shielding requirements for PET/CT. The EANM recommended clinical protocol for PET/CT examinations was used in order to determine how patients and staff will move within the department such that clinical operations are effective and radiation exposure is minimized [3].

\subsection{Procurement}

The decision to purchase a PET/CT required a procurement process. A multidisciplinary team from 
the hospital had to evaluate the characteristics of PET/CT machines and accompanying service contracts that were offered by three different vendors. The team included a medical physicist, medical technical personnel, nuclear medicine staff, and construction project personnel.

The evaluation of offers from the vendors was accomplished by using a specification of requirements. The specification was a list of features of the PET/CT, including details about the physics of the PET, physics of the CT, hardware, software, IT-solutions, energy use, heating/cooling requirements, physical layout and size, options, and service contracts. The specification was independent of vendor and included a grading of how important each feature was to the hospital, that is, whether a feature was obligatory, desired, or optional. Each vendor replied independently to the specification by describing how their offer of PET/CT and service fulfilled the requirements listed.

The vendors and PET/CT machines under consideration for Østfold Hospital Kalnes were the GE Discovery 610, Siemens Biograph mCT, and Philips Vereos PET/CT.

The procurement team studied literature and materials provided by the manufacturers and visited clinical installations of each PET/CT machine with a vendor representative. Each member of the team focused on the particular details of the specification of requirements that matched his field of expertise. The physics of the PET components available from each vendor, for example, were evaluated by the medical physicist, while personnel from the medical technical department assessed the cooling requirements and energy consumption of each PET/CT system.

\subsection{Training}

Clinical operations of a PET/CT can be effective only in relation to the competence of those nuclear medicine personnel who will use the machine. The nuclear medicine staff of Østfold Hospital was familiar with clinical SPECT/CT, but nobody had worked in a department with PET/CT. In order to determine how best to prepare a SPECT/CT staff for clinical implementation of PET/CT, a questionnaire was sent to hospitals in Norway that have clinical PET/CT in clinical use. Training activities for the medical physicist included courses and study visits to other hospitals. Training for the nuclear medicine staff included department seminars given by the physicist. Staff members who visited other hospitals in order to observe clinical routines and quality control procedures also shared information with colleagues during department meetings.

\section{RESULtS}

\subsection{Shielding the Nuclear Medicine Department}

The nuclear medicine department at Østfold Hospital Kalnes opened in May 2015 with a room that had been constructed for SPECT/CT adequately shielded for clinical PET/CT examinations. In addition, three patient uptake rooms had been designated, along with an area assigned for clinical operations of an automatic dose injector. The layout of the nuclear medicine department is shown in Figure 1, with the newly designated PET/CT room marked. All PET patient rooms are gathered to one half of the department, with a low occupancy corridor on the opposing side of the department. If PET patients are not able to leave the hospital immediately after their procedure, it may be possible for them to wait in this corridor in order to reduce exposure to nuclear medicine staff, other patients, and the public.

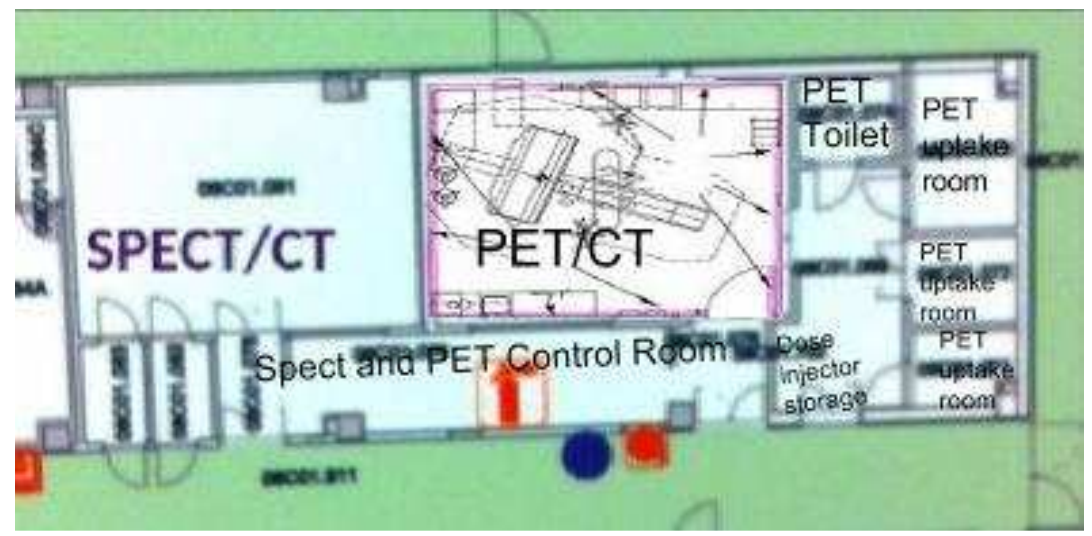

Figure 1. Layout of the nuclear medicine department at Østfold Hospital Kalnes. One imaging room accommodates a SPECT/CT, and the other imaging room was modified in order to install a clinical PET/CT

When building for SPECT/CT, 2mm lead walls and doors were installed. While this is sufficient for SPECT/CT, it is not sufficient for clinical PET/CT. The half value layer for $511 \mathrm{keV}$ annihilation radiation photons is $4 \mathrm{~mm}$ lead in a narrow beam geometry, and $5 \mathrm{~mm}$ for broad beam. A shielding of $1.6 \mathrm{~mm}$ lead, which is sufficient for CT radiation, provides a transmission factor of 0.81 for the annihilation radiation of PET [4].
For concrete, a standard building material, the half value layer thickness for $511 \mathrm{keV}$ photons is $3.4 \mathrm{~cm}$. A wall or floor of $10 \mathrm{~cm}$ concrete will therefore provide a dose reduction factor of 2.5 for PET annihilation radiation [4]. Lead shielding was added to the walls and doors of the room that would accommodate the new PET/CT. In addition to the concrete in the walls and floors, 4mm lead shielding surrounds the PET/CT 
imaging room. Each of the three patient uptake rooms is shielded by a $3 \mathrm{~mm}$ lead door. In addition, the window to the control room is leaded glass. The standard for a shielded viewing window to a PET control station is an $8 \mathrm{~mm}$ leaded glass window.

A patient toilet designated exclusively for PET patients was established adjacent to the patient uptake rooms and workspace for the automatic dose injector. Aside from the $4 \mathrm{~mm}$ lead door between the PET/CT imaging room and the staff control room, there is only one, shielded entrance to the workspace area that grants access to the patient uptake rooms, dose injector storage, and PET patient toilet. The patient toilet that is reserved for PET patients is located in the area of the uptake rooms, and is separated from the SPECT/CT in the department so as to avoid altering the background counts of other detectors in the department.

A delay tank from the PET/CT patient toilet to the basement of the hospital was installed. During full clinical operations, the tank will empty once per 24hours. A physicist made calculations of the estimated accumulation of radioactivity to the delay tank and release of radioactivity to the county sewage system during full clinical operations. The calculations were sent to the national radiation protection authority and the local county, as required for approval prior to clinical operation of PET/CT.

It is noted that the calculations were based on an estimated full clinical activity, 8 patients per day and an FDG injected activity of $340 \mathrm{MBq}$ per patient, but this will not be achieved immediately. The department intends to start conservatively, with probably two patients per week for a period of at least a few months, perhaps a half year, before gradually coming into full clinical operation. This will allow time for the staff to become proficient in the execution of PET/CT examination protocols. Full clinical activity at Østfold Hospital Kalnes will be limited by the availability of FDG from Oslo, where it is currently produced only once per day. In addition, image quality studies under the first months of operation may lead to a lower injected activity per patient.

In order to achieve greater radiation protection for the staff, the decision was made to use an automatic dose injector as part of the routine clinical PET/CT operations. The inclusion of an automatic dose injector was requested in the PET/CT specification of requirements that was sent to all vendors, meaning that all vendors had to specify an automatic dose injector and price for it in their PET/CT offer. The dose injector spares radiation to the staff, although not entirely. Some exposure will occur when the FDG vial is inserted or exchanged in the dose injector, and residual FDG in the tubes that deliver radioactivity to the patient may contribute to exposure of the staff when changing the tubes for each patient. These exposures are, in total, less than the exposure of manual preparation of each syringe and manual injection.

\subsection{Selecting the PET/CT system}

After evaluating all elements of the specification of requirements, the procurement team decided to purchase the Philips Vereos digital PET/CT and the Medrad Intego dose injector. In accordance with the specification of requirements, the scanner can perform all types of PET examinations and comes with protocols and equipment for performing all scan types.

Technology of interest in the Vereos PET/CT includes the detector crystals. Melcher describes properties and corresponding purposes of the ideal scintillation crystal for PET, including high density and atomic number for highest gamma ray detection efficiency [5]. LYSO crystals have high light output, and the $4 \mathrm{~mm} \times 4 \mathrm{~mm} \times 19 \mathrm{~mm}$ crystal size on the Vereos system are the smallest available. Smaller crystals improve spatial resolution via a narrow Full Width Half Maximum. Digital photon counting technology eliminates Photomultiplier-tubes. Crystals and detector elements are coupled 1:1 for improved spatial resolution.

In addition, the scanner has Time of Flight, a technological innovation that brings coincidence timing resolution to the imaging process. The coincidence timing information reduces the uncertainty or spread of information, yielding images with better signal to noise ratio. With Time of Flight, the difference in the arrival times of the two annihilation photons is measured with high precision that helps to localize the emission point along the line of response within a small region of the object. The uncertainty in this localization is determined by the system coincidence timing resolution, which is measured as the Full Width Half Maximum of the histogram of Time of Flight measurements from a point source, that is to say, the timing spectrum [6]. The Vereos PET/CT has a timing resolution of 345 picoseconds and a timing window of 4 nanoseconds.

Table 1 compares the crystal sizes and Photomultiplier tube coupling solutions from each of the PET/CT systems under consideration for Østfold Hospital Kalnes. GE refers to the Discovery 610, Siemens is the Biograph $\mathrm{mCT}$, and Philips Vereos PET/CT. The timing resolution of each PET/CT system is listed in Table 1. 
Table 1. Physical properties of PET/CT from each of the systems under consideration for Østfold Hospital Kalnes. GE refers to the Discovery 610, Siemens is the Biograph mCT, and Philips Vereos PET/CT

\begin{tabular}{|c|c|c|c|c|c|c|c|c|}
\hline \multicolumn{9}{|c|}{ Physical Properties of PET/CT } \\
\hline Manufacturer & $\begin{array}{l}\text { Detector } \\
\text { technology }\end{array}$ & $\begin{array}{c}\text { Crystal size } \\
\text { (mm) }\end{array}$ & $\begin{array}{l}\text { \#crystals per } \\
\text { detector }\end{array}$ & $\begin{array}{l}\text { Timing } \\
\text { resolution } \\
\text { (ps) }\end{array}$ & $\begin{array}{l}\text { Coincidence } \\
\text { window (ns) }\end{array}$ & Peak NECR & $\begin{array}{l}\text { Transaxial FOV, } \\
\text { "bore diameter" } \\
\text { (cm) }\end{array}$ & $\begin{array}{l}\text { Scatter } \\
\text { fraction } \\
(\%)\end{array}$ \\
\hline GE & Analogue & $4,7 \times 6,3 \times 30$ & $\begin{array}{l}\text { 12crystals/ } \\
\text { PM tube }\end{array}$ & 550 & 9,5 & 76kcps@15kBq/mL & 70 & $38 \%$ \\
\hline Siemens & Analogue & $4 \times 4 \times 20$ & $\begin{array}{l}\text { 42crystals/ } \\
\text { PM tube }\end{array}$ & 540 & 4,1 & 175kcps@ $\leq 28 \mathrm{kBq} / \mathrm{mL}$ & 78 & $38 \%$ \\
\hline Philips & Digital & $4 \times 4 \times 19$ & $\begin{array}{c}\text { 1:1 coupling } \\
(23,040 \\
\text { digital } \\
\text { detectors })\end{array}$ & 345 & 4,0 & 650kcps@50kBq/mL & 67,6 & $31 \%$ \\
\hline
\end{tabular}

Another technological specification of interest when choosing a PET/CT system is the axial detector width. A wide detector in the axial direction will allow scanning a given patient volume with fewer bed stops, thus contributing to faster examinations, to the benefit of patients and clinical operation. With the True V additional detector ring, Siemens Biograph mCT has 4 detector rings and the widest axial field of view, $21.6 \mathrm{~cm}$. GE Discovery 610 also has 4 detector rings, and the smallest axial field of view of the systems considered, $15.7 \mathrm{~cm}$. Philips Vereos lands between these extremes, with a $16.4 \mathrm{~cm}$ axial field of view and 5 detector rings.

The PET/CT signal is known to degrade from the center to the edge of the axial field of view. Bed overlap is a characteristic also of interest when choosing a PET/CT system since overlapping sequential bed positions compensates for signal degradation. Axially varying noise sensitivity may result from insufficient bed overlap. The Philips Vereos is optimized with a fixed bed overlap of $32 \%$. GE Discovery 610 has a standard overlap of $23 \%$ that corresponds to 11 slices and can be adjusted with respect to between one and 23 slices. Siemens Biograph mCT is optimized with a bed overlap of $43 \%$ or comes with the options of FlowMotion technology, in which the patient couch moves continuously during the scan. With FlowMotion technology, the speed of the couch movement can be adjusted as a type of parallel to changing bed overlap.

One method of comparing system performance is to look at the noise equivalent count rate and scatter fraction, summarized in Table 1 for the three PET/CT systems under consideration. The noise equivalent count rate describes the effective number of counts measured as a function of activity in the field of view. Image noise can be minimized by maximizing the noise equivalent count rate. A lower scatter fraction leads to better scanner performance and better image quality.

In addition to selecting a PET/CT based only on physical data in a general specification of requirements, the staff who will use the machine must be taken into account. One advantage of choosing the Vereos system for the staff at Østfold Hospital is that the radiology department already has a Philips Ingenuity CT. Protocols from the Ingenuity CT at the hospital can be imported to the new PET/CT, and radiology staff who work with the Ingenuity CT can easily perform CT examination with the Vereos. Indeed, it is of interest to the hospital to use the CT of the Vereos independently of the PET in addition to combined PET/CT examinations. This makes the purchase more effective, as the CT can be used during hours when PET/CT patients are not scheduled. Training on the CT part of the Vereos is also easier since radiologists are already familiar with the Ingenuity CT workstation.

The new Østfold Hospital Kalnes was planned for two SPECT/CT machines, therefore obviously without a thought to a medical cyclotron. It was not within budget, time, or physical space to construct a medical cyclotron facility at the new hospital, yet clinical operation of PET/CT relies on the availability of positron emitting radionuclides. Without a cyclotron, clinical examinations must use F-18 based radiotracers coming from medical cyclotrons in Oslo, which is about one hour by car from Østfold Hospital Kalnes. In the future, generator produced positron emitting radioisotopes may be used. This limits clinical operations to those exams where $\mathrm{F}-18$ based traces are effective.

\subsection{Training the Nuclear Medicine Staff}

Theoretical training of the nuclear medicine staff was accomplished by internal seminars and study visits to other hospitals. Department seminars highlighted radiation safety and clinical PET/CT protocols, and visits to other hospitals were made in order to observe clinical routines and quality control procedures. Practical training on the specific PET/CT equipment that was purchased, including training to use the automatic dose injector, took place at the time of installation in November 2015.

In addition to passive TLD dosimeters, staff members will wear electronic personal dosimeters and ring dosimeters, at least during the initial months of clinical PET/CT operations. After the nuclear medicine staff members are familiar with PET/CT examination procedures, it may be possible to eliminate the ring dosimeters. The measurements from all types of dosimeters will be studied over time to determine if the staff can safely work without, for example, ring dosimeters. This will save costs of maintaining and reading the ring dosimeters, and replacing the rings that may easily be lost. In addition, it is always possible to periodically use ring dosimeters again and study the results for a period of time, or to reserve them for new staff members in training.

Clinical workflow was learned by visiting other hospitals and reading the current EANM guidelines for FDG clinical PET/CT studies [3]. Included in the 
clinical workflow is glucose measurement of each patient prior to injection of FDG. If the plasma glucose level is lower than $11 \mathrm{mmol} / \mathrm{L}$, the FDG PET/CT examination will be performed [3]. Windows to the injection rooms as well as to the scanning room permit visual patient monitoring by the staff, in addition to alarms in each uptake room that a patient may activate as necessary.

Quality control tests were also learned by visiting other hospitals and studying the literature, including recommendations from the vendor. Protocols for clinical examinations were written based on the EANM guidelines and protocols used by other hospitals in Norway. These protocols address patient positioning for acquisition, acquisition planning, and imaging and process control. Generally, the patient should be positioned with the arms elevated and supported above the head in order to avoid beam-hardening artefacts in the abdominal and pelvic regions, as well as artefacts caused by truncation of the measured field of view [3]. The examination protocol consists of a scout scan and a low dose CT for attenuation correction and anatomical correlation.

Since an automatic dose injector will be used, the preparation of radiopharmaceutical doses will be learned when the vendor trains the staff to use the automatic dose injector. The staff is already accustomed to radiopharmaceutical preparation for SPECT, and these skills can be applied to the preparation of PET syringes in a specially shielded PET hotlab, as an alternative to using the automatic dose injector.

Rotation of staff members among the various work stations of PET/CT is also planned in order to minimize the annual dose to each member of the department. Staff members will not be assigned to a particular station, such as the injection of patients or the control room, but will rotate periodically in order for each member of the staff to become proficient with each step of the PET/CT examination and to spare radiation exposure to individual employees.

\section{DISCUSSION}

The opening of a new hospital facility is an exciting event, and Østfold Hospital Kalnes opened a modern facility that includes PET/CT in the nuclear medicine department.

Offering PET/CT examinations enhances the diagnostic possibilities that available to patients in the region, and contributes to the growth of PET/CT in Norway.

Since Østfold Hospital Kalnes is not a university hospital and is completely new to PET/CT, it was decided to join the EARL FDG quality assurance program from the European Association of Nuclear Medicine [7]. Participation in EARL will enhance confidence of both the staff and the public because accreditation shows that the department performs PET/CT studies at a level that is comparable to university hospitals, both in Norway and other European countries, that have a long clinical history with clinical PET/CT. The program will also increase possibilities for collaboration and research studies.

The use of a dose injector demonstrates attention to radiation safety of the nuclear medicine staff. The installation of a modern, digital machine and participation in the EARL FDG quality assurance program expand research possibilities for Østfold Hospital Kalnes, attracting collaborators and providing the most modern job training experience for workers in the nuclear medicine department. As more digital PET/CT machines are installed in Europe, the staff at Østfold Hospital Kalnes will be able to serve as mentors who educate colleagues in the use of digital technology and the interpretation of imaging results from the Vereos PET/CT.

Acknowledgement. The content of this work is solely the responsibility of the author, who is not currently employed by or affiliated with Østfold Hospital at the time of publication of this work.

\section{REFERENCES}

1. M.T. Madsen et al., "AAPM Task Report 108: PET and PET/CT Shielding Requirements," Med. Phys., vol. 33, no. 1, pp. 4-15, Jan. 2006

2. "Safety Reports Series No. 58. Radiation Protection in Newer Medical Imaging Techniques: PET/CT,” IAEA, Vienna, Austria, 2008

3. R. Boellaard et al., "FDG PET and PET/CT: EANM Procedure Guidelines for Tumour PET Imaging: Version 2.0," Eur. J. Nucl. Med. Mol. Imag., vol. 37, no.2, pp. 328-354, Feb. 2015

4. J.A. Cruzate and A.P. Discacciatti, "Shielding of Medical Facilities, Shielding Design Considerations for PET-CT Facilities," Proc. 12th Congr. IRPA, Buenos Aires, Argentina, 2008

Retrieved from:

http://www.irpa12.org.ar/PDF/RC/RC 18 fullpaper.pd f 4

5. C.L. Melcher, "Scintillation Crystals for PET," J. Nucl. Med, vol. 41, no. 6, pp. 1051-1055, 2000

6. S. Surti, "Update on Time-of-Flight PET Imaging," $J$. Nucl. Med., vol. 56, no. 1, pp. 98-105, Jan. 2015

7. Manual for EARL FDG-PET/CT Accreditation, ver 2.1.b, Research 4 Life, an EANM Initiative, Mar. 2014

8. S. Ivanova, A. Artinyan, "Calculations and Analysis of the Radiation Protection of PET/CT Center," BgNS TRANSAC., vol. 20, no. 1, pp. 54-57, 2015 